\title{
变形图驱动和变形感知的谱姿态迁移
}

\author{
邹歆仪 ${ }^{1)}$, 李桂清 ${ }^{1)^{*}}$, 尹梦晓 ${ }^{2)}$, 柳雨新 ${ }^{1)}$, 王宇攀 3 ) \\ ${ }^{1)}$ (华南理工大学计算机科学与工程学院 广州 510006) \\ 2) (广西大学计算机与电子信息学院 南宁 530004) \\ 3) (网易(杭州)网络有限公司 杭州 310052) \\ (ligq@scut.edu.cn)
}

\begin{abstract}
摘 要: 为了提高姿态迁移过程中网格表面形状细节的保持能力, 减少分层姿态迁移的交互环节, 提出变形图驱动 且变形感知的自动分层谱姿态迁移方法. 首先利用变形图对三维模型进行形状保持的全局低频姿态迁移; 然后根据 模型变形前后的特征变化自动分割出局部刚性块, 并对它再次进行姿态迁移, 直到所有局部网格的姿态得到充分迁 移. 通过多个例子对文中方法和 Yin 等方法进行了比较, 实验结果表明, 文中方法次级姿态迁移的次数降低 $38.0 \%$, 平均距离误差降低 $54.0 \%$, 表面积和体积的变化降低 $12.5 \%$. 该方法的姿态迁移较充分, 在模型的形状保持上更有优 势, 且自动化程度较高.
\end{abstract}

关键词：姿态迁移; 网格简化; 变形图; 网格分割

中图法分类号: TP391.41 DOI: 10.3724/SP.J.1089.2021.18667

\section{Deformation-Graph-Driven and Deformation Aware Spectral Pose Transfer}

\author{
Zou Xinyi ${ }^{1)}$, Li Guiqing ${ }^{1)^{*}}$, Yin Mengxiao ${ }^{2)}$, Liu Yuxin ${ }^{1)}$, and Wang Yupan ${ }^{3)}$ \\ 1) (School of Computer Science \& Engineering, South China University of Technology, Guangzhou 510006) \\ 2) (School of Computer, Electronics and Information, Guangxi University, Nanning 530004) \\ 3) (Netease Cooperation, Hangzhou 310052)
}

\begin{abstract}
A deformation graph driven and deformation aware spectral pose transfer method is proposed in order to improve the ability of the spectral pose transfer method in preserving shape details and reducing user interactions. First, a deformation graph is introduced to drive the source model deforming under the guidance of low-frequency spectral coefficients of the reference model to obtain a deformed source model. Second, the deformed source model is automatically segmented to parts according to the deformation. Third, repeating the spectral pose transfer on two source parts separately yields two deformed source parts. This process can be repeated again if necessary. Finally assembling all deformed parts in a reversed order results in the completely deformed source model. Comparison with the method of Yin et al. is conducted via a couple of examples. Experimental results show that the secondary pose migrations are reduced by $38 \%$, the average distance error is reduced by $54 \%$, and both surface area and volume variations are reduced by about $12.5 \%$. It improves the quality of transfer results to a certain extent, reduces the number of middle-frequency pose transfer, and also reduces manual interaction.
\end{abstract}

收稿日期: 2020-09-15; 修回日期: 2020-11-16. 基金项目：国家自然科学基金(61972160, 61762007, 61572202). 邹歆仪(1994一), 女, 硕士, 主要研究方向为计算机图形学; 李桂清(1966-), 男, 博士, 教授, 博士生导师, CCF 高级会员, 论文通讯作者, 主要研究 方向为数字几何处理、三维动画、虚拟现实、图像与视频编辑等; 尹梦晓(1978一), 女, 博士, 副教授, 硕士生导师, 主要研究方向为 计算机图形学、数字几何处理; 柳雨新(1993-), 男, 博士研究生, 主要研究方向为计算机图形学; 王宇攀(1990一), 男, 博士研究生, 主要研究方向为计算机图形学. 
Key words: pose transfer; mesh simplification; deformation graph; mesh segmentation

三维网格模型的变形与编辑 ${ }^{[1]}$ 被广泛应用于 在计算机动画、影视、虚拟现实和三维游戏等领域. 但是，即使有了好的变形方法，通过交互的方式生 成新的姿态也仍然是既困难又耗时的事情, 因此 就有了基于样例(或数据驱动)的变形技术. 例如, 在计算机动画制作中，可以将参考模型的动画序 列迁移到目标模型上, 这就是变形迁移 ${ }^{[2-3]}$. 该技 术提取出参考序列相邻帧之间的三角形的几何变 换, 然后将其应用于目标模型, 产生类似的变形.

但很多情况下，由于没有运动序列，只是有 2 个姿态形状都不同的网格模型. 因此, 如何让它们 互相学习对方的姿态呢? 这就是姿态迁移 ${ }^{[4-5]}$ 所要 解决的问题. 较早的有 Levy ${ }^{[4]}$ 的平凡姿态迁移, 即 2 个给定的网格模型有相同的顶点数和连接关系. Kovnatsky 等 ${ }^{[5]}$ 提出的耦合准调基谱姿态迁移方法, 把姿态迁移推广到顶点数和边连接都不同的模型 之间的姿态学习. 但上述方法的细节保持能力较 差，而且姿态学习不充分. Yin 等 ${ }^{[6]}$ 提出的细节保持 的多层次谱姿态迁移方法, 很好地解决了上述问 题. 但其利用重心坐标子空间技术进行加速，因此 局部控制能力较差; 由于需要用户交互地指定并 分割需要进行次级姿态迁移的局部网格, 非常耗 时烦琐; 此外，分割下来的局部网格在姿态迁移 后, 重新拼接时一般需要调整朝向.

为此，本文通过分析模型顶点位置自动识别 变形区域并对其进行分割，以减少交互; 引人嵌人 变形图子空间来解决局部控制问题, 从而更好地 保持模型细节特征; 此外, 还优化了局部网格与整 体网格的拼接方法，以保证交界处的自然过渡。

\section{1 相关工作}

\section{1 网格变形}

网格变形是指给定一个三维网格模型及其顶 点子集的新位置或变换作为约束，构造一个既能 满足约束条件又能保持合理的几何或动力学特性 的新网格. 空间变形算法 ${ }^{[7]}$ 的思想是将物体嵌人一 个空间中, 通过控制空间的变形驱动物体的变形, 如自由变形算法 ${ }^{[8]}$ 是利用三维栅格对模型进行变 形，但是大部分情况下栅格无法很好地逼近物体 的形状, 这种方法只能得到粗略的变形结果, 而不 能保证局部变形的质量. 文献[9]提出了变形图的 概念，对模型表面采样得到一个粗䊁的控制网格，
称为变形图, 通过变形图节点的变化推导出原模 型形状的变化, 这种方法在变形过程中能够较好 地保持原始模型的形状特征. 在网格模型表面形 状特征的保持方面, 用拉普拉斯坐标保持网格模 型的几何细节 ${ }^{[10]}$, 用 ARAP(as rigid as possible)的 变形方法 ${ }^{[11]}$ 让网格模型中的单元尽可能地做刚性 变换, 以有效地保持模型局部的形状细节; 样例驱 动的方法 ${ }^{[12]}$ 在网格模型变形时最小化边长、二面角 和网格体积的变化程度.

\section{2 姿态迁移}

给定参考和源 2 个网格模型, 允许 2 个模型的 姿态和连接关系不相同, 且没有对应关系, 姿态迁 移要解决的问题是将源模型变成与参考模型具有 相似姿态的新模型, 不要求参考模型的变形过程 已知. Levy ${ }^{[4]}$ 提出一种平凡的谱姿态迁移方法, 把 网格的形状空间投影到拉普拉斯矩阵的调和基上. 基于低频基和高频基对应的系数分别反映形状的 姿态和细节特性, 通过交换 2 个具有相同连接关系 网格的低频系数, 实现粗踈的姿态迁移. 但其使用 的拉普拉斯特征基在不等距情况下的表达会有很 大差别, 导致姿态迁移结果存在严重的扭曲现象, 且姿态学习不充分.

为此, Kovnatsky 等 ${ }^{[5]}$ 提出了耦合准调和基的 概念，构造了 2 个不等距模型之间的基于泛函映射 的兼容的特征基, 但仍会出现细节丢失的问题. 这 2 种方法都是通过简单的系数交换进行姿态迁移, 容易丢失细节且次级姿态迁移不充分.

Yin 等 ${ }^{[6]}$ 提出一种细节保持的多层次谱姿态迁 移方法：在文献 [5]的基础上，通过保持在旋转意 义下的拉普拉斯坐标防止细节丢失; 在求解时用 基于均值坐标的子空间技术降低解空间的规模; 同时构建了一个分层谱姿态迁移框架，以保证中 尺度的姿态得到良好的迁移. 由于广义重心坐标 不满足局部性，该方法的结果受控制网格质量的 影响较大. 此外, 该方法完全由人工指定并分割需 要进行次级姿态迁移的局部网格.

文献[13]利用嵌人变形算法对文献[6]的谱姿 态迁移结果进行优化. 文献[14]将基于耦合准调和 基的谱姿态迁移方法应用于四边形网格. 文献[15] 将问题进一步扩展到四边形网格与三角网格间的 姿态迁移上. 文献[16]用基于局部坐标的拉普拉斯 偏移量驱动人体模型向模板姿态变形实现姿态迁 移. 文献[17]先将参考模型的表面形状变形到与源 
模型一致后，再将问题转化为 2 个姿态相同的模型 之间的形状迁移，但是该方法要求参考模型有一 个与源模型相同的初始姿态.

\section{3 网格分割}

三维网格模型的分割是指根据一定的几何和 拓扑特征对网格的顶点或面进行分类, 通常先计 算网格的特征描述子，然后利用这些特征对顶点 或者面进行相似性度量, 最后选择合适的分割算 法并定义分割准则, 以实现网格的分割.

关于单个网格分割的研究非常丰富, 分割的 本质是一个聚类问题, 经典的三维网格分割算法 有区域增长法、谱分析法、分层聚类法和骨架抽取 法等. 基于模糊聚类和最小割的层次分割法 ${ }^{[18]}$ 首 先用网格面之间的测地距离和角度距离作为特征 对网格进行粗聚类, 然后利用最小割算法 ${ }^{[19]}$ 在模 糊区域中寻找准确的分割边界. 基于谱聚类的分 割方法 ${ }^{[20]}$ 利用相邻面片差异性的度量 ${ }^{[18]}$ 构建一个 相似矩阵，然后用相似矩阵的特征向量将原始空 间中的数据映射到谱空间，并在谱空间中进行 $k$-means 聚类，以实现分割. 还有一类方法通过交 互的方式引人用户的分割意图, 文献[21]最早提出 用笔画交互指定前景和背景，然后基于改进的等 光距离用区域增长法将没有标记的区域进行标记.

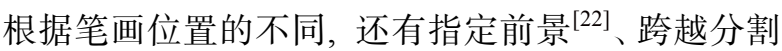
边界 ${ }^{[23]}$ 、沿着分割边界 ${ }^{[24]}$ 的方法, 文献[25]对这些 交互分割的方法进行了详细的说明.

对于运动物体的分割是根据变换类型把具有 相似变换的联通区域聚成一类，得到运动序列中 所有模型的一致分割. 其中, 刚性运动分割把模型 分成多个刚性运动区域，分割边界位于模型的关 节处. 基于网格动画中旋转变换相似的面构成一 个近似刚性的区域这一思想, 文献[26-27]对运动 序列中每个面的运动轨迹的变换矩阵进行聚类, 以实现运动序列的刚性共分割. 文献[28]先在参考 姿态上选取一些种子点, 并利用谱分割算法对这 些点的运动进行分类, 然后把种子点的分割结果 扩散到网格所有的面上. 文献[29-30]用骨架蒙皮 来表示相同拓扑的网格序列, 并对顶点的运动轨 迹进行 $k$-means 聚类. 文献[31]构造了一个模板运 动序列, 用它去拟合输人数据, 然后用自适应的谱 聚类方法对顶点的刚性变换矩阵进行聚类. 文献 [32]利用二面角的变化度量局部变形程度, 通过最 大化类间距离实现网格分割. 以上方法对于关节 运动物体的分割效果都比较理想, 但均需要一个 连续的运动序列作为输人, 而本文仅有 2 个模型,
虽然对它们进行插值也能得到运动序列, 但这意 味着需要添加额外的步骤.

\section{2 变形图驱动的谱姿态迁移}

\section{1 姿态迁移的问题描述}

给定 2 个三角网格模型：源模型 $M=\langle\boldsymbol{V}, \boldsymbol{E}, \boldsymbol{F}\rangle$, 参考模型 $M^{\prime}=\left\langle\boldsymbol{V}^{\prime}, \boldsymbol{E}^{\prime}, \boldsymbol{F}^{\prime}\right\rangle$. 其中, $\boldsymbol{V}$ 为顶点的集合 $\boldsymbol{V}=\left\{\boldsymbol{v}_{i}, i=0,1, \cdots,|\boldsymbol{V}|-1\right\}$, 顶点的位置在三维欧几 里得空间中用三维坐标表示为 $\boldsymbol{v}_{i}=\left(x_{i} y_{i} z_{i}\right)^{\mathrm{T}} ; \boldsymbol{E}$ 和 $\boldsymbol{F}$ 分别表示边和面的集合, 模型 $M^{\prime}$ 同理. 姿态 迁移技术将 $M^{\prime}$ 的姿态迁移到 $M$ 上, 生成一个新 的模型 $\tilde{M}$, 称为目标模型, $\tilde{M}$ 由 $M$ 变形得到, 具 有与 $M^{\prime}$ 相似的姿态，且保持着 $M$ 的形状细节.

\section{2 谱姿态迁移}

网格 $M$ 的拉普拉斯矩阵定义为 $\boldsymbol{L}=\boldsymbol{D}^{-1} \boldsymbol{W}^{[4]}$, 其中, $\boldsymbol{D}^{-1}$ 是以顶点 Voronoi 面积的倒数作为元素 的对角阵, $W$ 是余切权重下的对称矩阵. 对拉普 拉斯矩阵做特征分解 $\boldsymbol{L}_{\phi_{1}}=\lambda_{i} \phi, i=0,1, \cdots,|\boldsymbol{V}|-1$, 得 到特征值 $\lambda_{i}$ 和特征向量 $\phi$. 将特征值及其对应的 特征向量按照特征值从小到大的顺序排序, 排序 后的特征值和特征向量分别称为拉普拉斯矩阵 $\boldsymbol{L}$ 的谱和网格 $M$ 的调和基, 则网格 $M$ 的顶点坐标可 以由调和基线性混合得到, 即 $\boldsymbol{V}=\sum_{i=0}^{|\boldsymbol{V}|-1} \alpha_{i} \boldsymbol{\phi}_{i}$; 其中, $\alpha_{i}$ 为谱系数. 较小特征值对应的谱系数称为低频 系数, 通常编码模型的整体姿态; 而较大特征值对 应的高频系数, 其反映模型的形状细节. 可以认 为, 整体姿态相似的模型拥有相近的低频系数. 基 于这一特性, Levy $y^{[4]}$ 把参考模型的低频系数复制到 源模型上实现姿态迁移，即目标模型 $\tilde{M}$ 的顶点坐 标为 $\tilde{\boldsymbol{V}}=\sum_{i=0}^{K} \alpha_{i}^{\prime} \boldsymbol{\phi}_{i}+\sum_{i=K+1}^{|\boldsymbol{V}|-1} \alpha_{i} \boldsymbol{\phi}_{\boldsymbol{l}}$. 但一般情况下, 源模型 和参考模型的调和基是不等距的，直接交换低频 系数会导致姿态迁移结果存在严重的扭曲现象. 为了解决这个问题, Kovnatsky 等 ${ }^{[5]}$ 优化调和基得 到等距的耦合准调和基. 网格变形应该是整体的、 联动的，而以上 2 种方法单纯地复制了参考模型的 低频系数, 没有考虑其他频率的系数也应该发生 一定的改变, 显而易见会出现细节丢失的问题. 为 此, Yin 等 ${ }^{[6]}$ 通过最小化低频能量

$$
E_{\text {freq }}=\sum_{i=0}^{K}\left\|\tilde{\boldsymbol{V}}^{\mathrm{T}} \boldsymbol{\phi}_{1}-\alpha_{i}^{\prime}\right\|^{2}
$$


实现姿态迁移，其作用是保证目标网格与参考网 格的前 $K$ 个低频系数相等, $K=5$. 此外, 还通过 保持拉普拉斯坐标来防止模型细节丢失, 并约束 模型各部分的均匀缩放.

\section{3 基于变形图的谱姿态迁移优化求解}

为了更好地保持网格的表面形状，本文使用 具有局部控制能力的嵌人变形图 ${ }^{[9]}$ 的子空间编辑 技术进行谱姿态迁移。首先用带三角形形状和面 积约束 ${ }^{[33]}$ 的二次误差度量 (quadratic error measure, QEM)算法 ${ }^{[34]}$ 对源网格进行简化，用简化网格构造 变形图; 然后根据式(1)的低频能量约束求出变形 图节点的变换，同时用局部刚性变换约束和相邻 点变化一致性约束保证保持模型的表面形状; 最 后线性组合这些变换, 以得到目标网格的顶点坐 标，进而实现姿态迁移.

记变形图 $G$ 的顶点集为 $\boldsymbol{V}_{G}$, 式(1)中目标网格

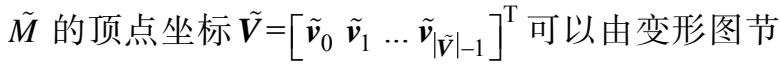
点的仿射变换线性混合得到 ${ }^{[9]}$, 即

$$
\tilde{\boldsymbol{v}}_{i}=\sum_{j=0}^{m-1} w_{j i}\left[\boldsymbol{R}_{j}\left(\boldsymbol{v}_{i}-\boldsymbol{g}_{j}\right)+\boldsymbol{g}_{j}+\boldsymbol{t}_{j}\right]
$$

其中, $\boldsymbol{g}_{j}$ 为变形图上距离源网格顶点 $v_{i}$ 测地线距 离最近的 $m$ 个节点, 每个 $\boldsymbol{g}_{j}$ 对应一个旋转矩阵 $\boldsymbol{R}_{j}$ 和平移向量 $\boldsymbol{t}_{j} ; w_{j i}=\left(1-\left\|\boldsymbol{v}_{i}-\boldsymbol{g}_{j}\right\| / d_{\text {max }}\right)^{2}$ 为 $\boldsymbol{g}_{j}$ 相对 于 $v_{i}$ 的控制权重，该权重与测地线距离相关, $d_{\text {max }}$ 为距离 $\boldsymbol{v}_{i}$ 第 $m+1$ 近的变形图节点到 $\boldsymbol{v}_{i}$ 的测地 线距离, 本文实验中 $m=4$.

单纯最小化式(1)的能量可能会导致过约束, 使网格产生不自然的变形. 为了保持模型的表面 形状，变形图节点的旋转矩阵应为 $S(3)$ 空间下的 旋转. 此时局部变换为刚性变换, 则旋转矩阵需满 足 2 个条件: 每个列向量均为单位向量, 且两两正 交. 记 $\boldsymbol{R}=\left[\begin{array}{lll}\boldsymbol{c}_{1} & \boldsymbol{c}_{2} & \boldsymbol{c}_{3}\end{array}\right]$, 上述条件的平方差为

$$
\begin{aligned}
R(\boldsymbol{R})= & \left(\boldsymbol{c}_{1} \cdot \boldsymbol{c}_{2}\right)^{2}+\left(\boldsymbol{c}_{1} \cdot \boldsymbol{c}_{3}\right)^{2}+\left(\boldsymbol{c}_{2} \cdot \boldsymbol{c}_{3}\right)^{2}+\left(\boldsymbol{c}_{1} \cdot \boldsymbol{c}_{1}-1\right)^{2}+ \\
& \left(\boldsymbol{c}_{2} \cdot \boldsymbol{c}_{2}-1\right)^{2}+\left(\boldsymbol{c}_{3} \cdot \boldsymbol{c}_{3}-1\right)^{2} .
\end{aligned}
$$

将变形图所有节点的旋转误差相加, 得到旋转约 束项 ${ }^{[9]}$ 为

$$
E_{\text {rot }}=\sum_{j=0}^{\left|V_{G}\right|-1} R\left(\boldsymbol{R}_{j}\right)
$$

此外, 每个仿射变换代表一个以变形图节点 为中心的局部变形. 由于相邻的变换具有重叠的 影响，因此必须确保计算出的变换彼此之间是一 致的. 如果节点 $\boldsymbol{g}_{j}$ 和 $\boldsymbol{g}_{k}$ 是邻居点, 那么通过 $\boldsymbol{g}_{j}$ 预
测出的 $\boldsymbol{g}_{k}$ 的新位置应与 $\boldsymbol{g}_{k}$ 应用自身的仿射变换得 到的位置相一致. 将这 2 个位置之间的平方距离求 和, 得到正则化误差 ${ }^{[9]}$ 为

$$
\begin{aligned}
& E_{\mathrm{reg}}= \\
& \sum_{j=0}^{\left|\boldsymbol{v}_{G}\right|-1} \sum_{k \in N(j)} \alpha_{j k}\left\|\boldsymbol{R}_{j}\left(\boldsymbol{g}_{k}-\boldsymbol{g}_{j}\right)+\boldsymbol{g}_{j}+\boldsymbol{t}_{j}-\left(\boldsymbol{g}_{k}+\boldsymbol{t}_{k}\right)\right\|_{2}^{2}
\end{aligned}
$$

其中, $N(j)$ 为节点 $\boldsymbol{g}_{j}$ 的邻域点集; 实验中取权重 $\alpha_{j k}=1$. 最终变形图驱动的谱姿态迁移问题转化为 求解化问题

$$
\min _{\boldsymbol{R}_{0}, \boldsymbol{t}_{0}, \cdots, \boldsymbol{R}_{V_{G} \backslash-1}, t_{V_{G} \mid-1}} w_{\text {freq }} E_{\text {freq }}+w_{\text {rot }} E_{\text {rot }}+w_{\text {reg }} E_{\text {reg }}
$$

其中, $E_{\text {freq }}, E_{\text {rot }}$ 和 $E_{\text {reg }}$ 计算公式分别如式(1)(3)(4) 所示, 那么待求的未知量为变形图所有节点的旋 转矩阵 $\boldsymbol{R}$ 和平移向量 $\boldsymbol{t}$, 本文使用高斯牛顿法优化 这个非线性最小二乘问题.

通过求解式(5), 可以得到具有与参考模型相似 的姿态, 并保持了源模型形状细节的目标模型. 本 文实验取权重 $w_{\text {freq }}, w_{\text {rot }}$ 和 $w_{\text {reg }}$ 分别为 $0.06,3.00$ 和 50.00. 如果目标模型的姿态或表面发生明显的变 形, 需要减小权重 $w_{\text {freq }}$; 反之, 若姿态迁移不到位, 则加大权重 $w_{\text {freq }}$ 或再对整体进行低频姿态迁移.

\section{4 低频姿态迁移结果}

图 1 图 3 所示为本文方法与 Yin 等 ${ }^{[6]}$ 方法对比 的结果.

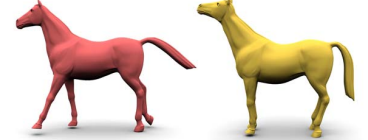

a. 参考模型 b. 源模型

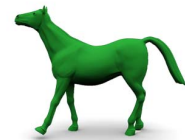

c. Yin 等 ${ }^{[6]}$

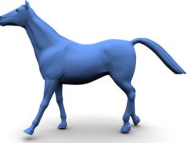

d. 本文
图 1 连接关系相同的同一对象之间的低频姿态迁移

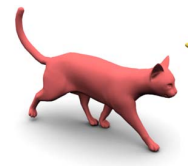

a. 参考模型

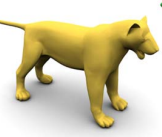

b. 源模型

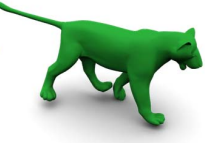

c. Yin 等 ${ }^{[6]}$

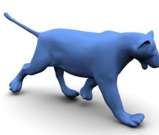

d. 本文
图 2 连接关系相同的不同对象之间的低频姿态迁移

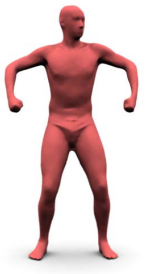

a. 参考模型

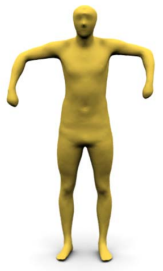

b. 源模型

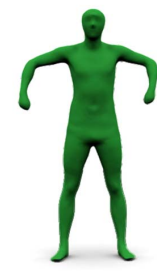

c. Yin 等 ${ }^{[6]}$

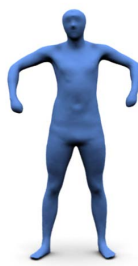

d. 本文
图 3 连接关系不同的人体模型之间的低频姿态迁移 
(1) 具有相同连接关系的同一对象

图 1 所示为一种最简单的情况, 具有相同连接 关系的同一模型不同姿态之间的低频姿态迁移. 可以看到，Yin 等 ${ }^{[6]}$ 方法结果无法捕捉到马的右后 腿、尾巴和头部的姿态; 而本文方法经过 2 次低频 姿态迁移后, 这些部位的姿态已经很接近参考模 型, 不必进行分层姿态迁移.

(2) 相同连接关系的不同对象

图 2 中的猫和狮子模型具有相同的连接关系, 与 Yin 等 ${ }^{[6]}$ 方法结果类似, 本文方法也能将参考模 型的大致姿态学习到位, 但尾巴的姿态未能充分 迁移. 但与 Yin 等 ${ }^{[6]}$ 方法结果相比, 本文方法的姿 态迁移更加到位，狮子的右前腿和右后腿伸展得 更远, 更接近参考模型的姿态, 而且尾巴可以向上 抬起一定的角度, 这为后续自动分层方法提供了 可能. 另外, 本文方法结果对于局部形状的保持效 果更好, 图 2c 中狮子的尾巴整体有了变粗的迹象, 而图 $2 \mathrm{~d}$ 中的尾巴形状仍与源模型保持一致.

(3) 不同连接关系的不同对象

图 3 所示为更一般的情况, 参考模型与源模型 的连接关系和形状都不相同. Yin 等 ${ }^{[6]}$ 方法和本文 方法都能学习到参考模型的整体姿态，而丢失了 头部和腿部这些局部的姿态, 但是本文方法结果 中人体的右手姿态已经迁移到位，与 Yin 等 $^{[6]}$ 方法 相比，可以减少一次分层姿态迁移的操作.

从以上结果可以看出, 本文基于变形图的低 频谱姿态迁移可以将参考模型的大致姿态迁移到 源模型上，由于引人了局部刚性变化和相邻点变 化一致性的约束，因此该方法能够保持模型的表 面细节和形状. 然而, 对于模型局部的姿态仍然没 有办法完全迁移到位, 需要进行分层姿态迁移, 但 不同于 Yin 等 ${ }^{[6]}$ 方法所使用的均值坐标, 变形图具 有局部控制能力, 每个顶点的变换只由以其为中 心的局部来控制, 而不会受到整体的影响, 因此部 分局部网格的姿态在这个阶段可以得到充分的迁 移, 这在一定程度上能够减少后续次级姿态迁移 的次数.

\section{3 变形感知与自动分层谱姿态迁移}

低频系数只能反映模型的整体特征, 而模型 的姿态具有多尺度性，Yin 等 ${ }^{[6]}$ 提出的分层姿态迁 移的框架在进行全局的低频姿态迁移后分割出局 部模型, 此时全局的中频姿态就转换为局部的低 频姿态，分割出所有姿态迁移不充分的局部模型
并对它们做姿态迁移, 直到所有部分的姿态都得 到充分的迁移. Yin 等 $^{[6]}$ 方法通过目测指定需要分 割的部位, 且所使用的简易分割算法需要在分割 处指定构成封闭回路的顶点集, 交互步骤多, 输人 要求高, 且部分局部模型在姿态迁移后需要进行 朝向调整以适应整体模型的朝向.

受文献[23]的启发, 本文提出一个自动分割局 部网格的方法. 首先计算拉普拉斯方程的边界约 束点, 然后求解带约束的拉普拉斯方程得到顶点 的调和场，根据调和场的值将模型粗略地分割为 3 个部分, 最后在模糊区域中利用最小割算法 ${ }^{[19]}$ 寻 找准确的分割边界. 同时, 用基于变形图的变形技 术引导局部模型的姿态迁移以及局部模型与整体 模型的拼接, 姿态迁移后的局部模型的形状得以 较好的保持, 不会发生整体朝向错误的情况, 且拼 接边界处能够自然过渡.

\section{1 变形感知与分割部位检测}

姿态迁移的对象多为人体、动物等带关节的模 型, 这些模型在姿态迁移时, 肢体部分的变形通常 为刚性运动, 分割局部模型的目标是把这些刚性 运动块分割出来, 使分割边界落在关节处. 根据低 频姿态迁移的结果可以发现这样一个规律: 低频 姿态迁移虽然不能准确捕捉到所有局部模型的姿 态, 但是这些局部模型也会发生一定程度的变形, 这为检测提供了可能, 本文按照局部模型变形程 度由大到小依次进行分割部位的检测和分割.

\subsection{1 计算变形特征}

本文用顶点位移量和三角面片的变形梯度衡 量模型的变形情况.

(1) 顶点位移量

顶点位移量是指目标模型的顶点与源模型上 对应点的欧几里得度量. 由于在变形过程中没有 对目标模型的位置做约束, 因此求解出来的目标 模型与源模型的位置和朝向可能存在差异. 为了 消除因为整体的平移和旋转对顶点位移量计算的 影响, 需要对源模型和目标模型做刚性对齐, 包括 平移和旋转 2 个操作. 用最远点采样算法 ${ }^{[35]}$ 选取模 型上的 30 个点, 根据这些点的平均坐标通过平移 操作对齐它们的位置, 通过最小化应用旋转矩阵 后的源模型与目标模型的距离的 Frobenius 范数计 算整体的旋转矩阵 ${ }^{[36]}$. 经过刚性对齐后, 计算每 个顶点 $v_{i}$ 的位移量

$$
D\left(\boldsymbol{v}_{i}\right)=\left|\boldsymbol{v}_{i}-\tilde{\boldsymbol{v}}_{i}\right|
$$

此时, 对于发生刚性运动的肢体部分, 顶点偏 移量的值在肢体末端最大, 往躯干方向逐渐减小. 


\section{(2) 变形梯度}

网格模型的变形可以看做每个三角形面片仿 射变换的结果 ${ }^{[2]}$, 仿射变换由带放缩的旋转矩阵 $\boldsymbol{Q}$ 和平移向量 $\boldsymbol{d}$ 构成. 由于 3 个顶点无法确定垂直 于三角形的空间如何变形，也就不能完全确定一 个三角形的仿射变换. 因此在垂直于三角形面片 的方向上定义 4 个顶点，这 4 个顶点按 $\tilde{\boldsymbol{v}}_{i}=\boldsymbol{Q} \boldsymbol{v}_{i}+\boldsymbol{d}$ ， $i=1, \cdots, 4$ 的方式变换，消去 $\boldsymbol{d}$ 后得到 $\tilde{\boldsymbol{V}}=\boldsymbol{Q} \boldsymbol{V}$. 其 中, $\tilde{\boldsymbol{V}}=\left[\tilde{\boldsymbol{v}}_{2}-\tilde{\boldsymbol{v}}_{1} \tilde{\boldsymbol{v}}_{3}-\tilde{\boldsymbol{v}}_{1} \tilde{\boldsymbol{v}}_{4}-\tilde{\boldsymbol{v}}_{1}\right] ; \boldsymbol{V}$ 同理, 则 $\boldsymbol{Q}$ 可以 近似为 $\boldsymbol{Q}=\tilde{\boldsymbol{V}} \boldsymbol{V}^{-1}$. 对 $\boldsymbol{Q}$ 进行右极分解，可以得到 消除放缩因子后的旋转矩阵 $\boldsymbol{R}$.

所有发生变形的面片的旋转矩阵都会发生变 化, 用旋转矩阵与单位矩阵之间的距离来衡量面片 的变化程度. 设 $3 \times 3$ 的单位矩阵为 $\boldsymbol{I}$, 记 $\boldsymbol{R}_{k}-\boldsymbol{I}=$ $\left[\begin{array}{lll}\boldsymbol{c}_{1}^{k} & \boldsymbol{c}_{2}^{k} & \boldsymbol{c}_{3}^{k}\end{array}\right], k=0,1, \cdots,|\boldsymbol{F}|-1$, 面 $\boldsymbol{f}_{k}$ 的旋转程度定 义为 $R\left(\boldsymbol{f}_{k}\right)=\left\|\boldsymbol{c}_{1}^{k}\right\|_{1}+\left\|\boldsymbol{c}_{2}^{k}\right\|_{1}+\left\|\boldsymbol{c}_{3}^{k}\right\|_{1}$, 顶点 $\boldsymbol{v}_{i}$ 的旋转程 度 $R\left(v_{i}\right)$ 取所在面的旋转程度的最大值, 即

$$
R\left(\boldsymbol{v}_{i}\right)=\max \left\{R\left(\boldsymbol{f}_{k}\right)\right\}, \boldsymbol{v}_{i} \in \boldsymbol{f}_{k}
$$

与顶点位移量类似，发生变形的肢体末端的 顶点旋转程度最大, 往躯干方向逐渐减小. 基于这 个规律，可以定位到变形肢体的关节位置区域.

\section{1 .2 边界约束点的选取}

结合顶点位移量和旋转程度定义顶点 $v_{i}$ 变形 程度的度量, 即

$$
M_{\mathrm{D}}\left(\boldsymbol{v}_{i}\right)=D\left(\boldsymbol{v}_{i}\right) R\left(\boldsymbol{v}_{i}\right)
$$

按式(8)计算出 $M_{\mathrm{D}}$ 值后, 将其归一化至 $[0,1]$. $M_{\mathrm{D}}$ 值的大小与变形前后顶点的变化程度正相关, 对于发生明显刚性运动的肢体, 其 $M_{\mathrm{D}}$ 值从肢体 末端往躯干方向逐渐减小，在关节处附近开始趋 向于 0 . 因此, 可以利用 $M_{\mathrm{D}}$ 值找到关节点的位置. 记 $M_{\mathrm{D}}$ 值最大的点为 $v_{\max }$, 在 $M_{\mathrm{D}}<\varepsilon$ (阈值)的顶 点中, 挑选距离 $\boldsymbol{v}_{\text {max }}$ 测地线距离最小的点为 $v_{\mathrm{key}}$, 则 $\boldsymbol{v}_{\mathrm{key}}$ 位于待分割局部网格的关节处附近. 实验 中, 一般取 $\varepsilon=0.1$. 然后, 从 $\boldsymbol{v}_{\mathrm{key}}$ 开始分别向 $M_{\mathrm{D}}$ 值最大和最小的方向搜索边界约束点. 具体的过 程为：从 $v_{\mathrm{key}}$ 开始，找到其 $M_{\mathrm{D}}$ 值最大的邻点; 然 后从该邻点出发, 再次找到 $M_{\mathrm{D}}$ 值最大的邻点, 重 复 $n$ 次这个过程; 最后一次选取的点即为 $\boldsymbol{v}_{p}$. 为 了避免出现回路，记录下经过的顶点下标，如果 $M_{\mathrm{D}}$ 值最大的邻点已经被选取, 那么就选次大值 的邻点. 同理，沿 $M_{\mathrm{D}}$ 最小的方向搜索 $n$ 次可以得 到点 $\boldsymbol{v}_{p}$. 实验中, 取 $n=5$.
由于需要将所有发生变形的局部网格检测并 分割出来, 因此在进行下一次分割位置检测时, 需 要剔除已经分割出来的局部网格. 本文分割位置 检测的方法是基于待分割的局部网格发生变形这 一前提, 若局部网格未发生变形或变形程度很小, 则无法检测, 需要人工判断该局部网格是否需要 分割, 以便进行次级姿态迁移; 若需要人工判断, 则指定期望的分割边界附近的 2 个顶点作为边界 约束点.

\section{2 局部网格的分割}

局部网格的分割分为粗分割和确定分割边界 2 个阶段, 在粗分割阶段, 根据文献[23]对调和场 的计算进行建模 $\min _{u\left(v_{0}\right), \cdots, u\left(\boldsymbol{v}_{|v|-1}\right)} E_{\text {lap }}+w_{p} E_{p}+w_{q} E_{q}$. 其 中, $E_{\text {lap }}=\|\boldsymbol{L} \boldsymbol{u}\|_{2}^{2} ; \boldsymbol{L}$ 为余切权重的拉普拉斯矩阵; 拉普拉斯方程 $\boldsymbol{L u}=\mathbf{0}$ 的解 $\boldsymbol{u}$ 称为调和函数. 通过求 解拉普拉斯方程可以得到一个光滑的调和场, 网格 上每个顶点 $\boldsymbol{v}_{i}$ 对应调和值 $u\left(v_{i}\right) . E_{p}=\left\|u\left(v_{p}\right)-1\right\|^{2}$ 和 $E_{q}=\left\|u\left(v_{q}\right)\right\|^{2}$ 为边界约束项, 用于指定分割边界 所在的区域, 使边界条件沿曲面扩散. 实验中, 取 $w_{p}=w_{q}=1$.

由于边界项的约束, $v_{p}$ 和 $v_{q}$ 之间的调和值在 0 1 的范围内, 而所希望的分割边界也落在这个区 域. 充分利用所求的调和值将模型粗略分为 3 个区 域：顶点集 $\boldsymbol{V}_{A}=\left\{\boldsymbol{v}, u(v)>1+\varepsilon_{1}\right\}$ 的区域 $A$; 顶点集 $\boldsymbol{V}_{B}=\left\{\boldsymbol{v}, u(\boldsymbol{v})<0+\varepsilon_{2}\right\}$ 的区域 $B$; 剩余的顶点归为第 3 类模糊区域 $C$, 顶点集记为 $\boldsymbol{V}_{C}$, 则所期望的分 割边界落在模糊区域 $C$ 中. 为了更准确地找到分 割边界，用 2 个國值增大模糊区域的范围，实验中， 即 $\varepsilon_{1}=0.3, \varepsilon_{2}=-0.5$. 若由于局部网格变形较小导 致选取的 $v_{q}$ 向远离躯的方向偏离关节处较远, 则适当减小 $\varepsilon_{2}$ 的值, 以保证模糊区域能够覆盖关 节位置.

在得到粗略的分割结果后，通过求解一个最 小割问题 ${ }^{[19]}$ 在模糊区域中寻找准确的边界. 在网 格 $M$ 上建立一个无向图 $G(M)=\langle\boldsymbol{V}, \boldsymbol{E}\rangle$, 网格的顶 点和边与无向图一一对应, 则网格分割问题可看 成一个贴标签问题, 目标是给每个顶点 $\boldsymbol{v}_{i} \in \boldsymbol{V}$ 分配 唯一的标签 $l\left(v_{i}\right) \in\{0,1\}$. 将粗分割结果 $\boldsymbol{V}_{A}$ 和 $\boldsymbol{V}_{B}$ 中 的顶点分别标记为 0 和 1 , 即 $l\left(v_{i}\right)=0, v_{i} \in V_{A}$, $l\left(v_{i}\right)=1, v_{i} \in V_{B}$, 则分割结果 $P=\left\{l\left(v_{i}\right)\right\}$ 可以通过 最小化能量 
$E_{\mathrm{gc}}=\sum_{\boldsymbol{v}_{i} \in \boldsymbol{V}} E_{\mathrm{data}}\left(l\left(\boldsymbol{v}_{i}\right)\right)+w \sum_{\left(\boldsymbol{v}_{i}, \boldsymbol{v}_{j}\right) \in \boldsymbol{E}} E_{\text {smo }}\left(l\left(\boldsymbol{v}_{i}\right), l\left(\boldsymbol{v}_{j}\right)\right)(9)$

得到. 其中, $E_{\mathrm{data}}$ 和 $E_{\mathrm{smo}}$ 分别为数据项和平滑项; $w$ 为平衡 2 个能量项重要程度的混合系数, 实验 中取 $w=1.5$.

数据项描述将标签 $l\left(v_{i}\right)$ 分配给顶点 $v_{i}$ 的代价, 定义为

$$
\begin{aligned}
& E_{\text {data }}\left(l\left(v_{i}\right)\right)= \\
& \left\{\begin{array}{lc}
0, & l\left(\boldsymbol{v}_{i}\right)=0, \boldsymbol{v}_{i} \in \boldsymbol{V}_{A} \text { or } l\left(\boldsymbol{v}_{i}\right)=1, \\
\infty, l\left(\boldsymbol{v}_{i} \in \boldsymbol{V}_{B}\right)=1, \boldsymbol{v}_{i} \in \boldsymbol{V}_{A} \text { or } l\left(\boldsymbol{v}_{i}\right)=0, & \boldsymbol{v}_{i} \in \boldsymbol{V}_{B} \\
\exp \left(\left|\boldsymbol{v}_{i}-\boldsymbol{v}_{l\left(\boldsymbol{v}_{i}\right)}\right|\right), & \text { 其他 }
\end{array}\right.
\end{aligned}
$$

其中, $v_{l\left(v_{i}\right)}$ 为标签为 $l\left(v_{i}\right)$ 的顶点的平均坐标. 对 于区域 $A$ 和区域 $B$ 中的顶点, 希望其标签不再变 化. 为简单起见, 若点 $v_{i} \in V_{A}$ 落在标签为 1 的区域 中，该点的数据项取无穷大; 若落在标签为 0 的区 域中, 数据项值为 0 , 区域 $B$ 中的点同理.

平滑项用于定义相邻 2 点间的相似性. 期望分 割边界位于关节处，这些位置的表面凹特征比较 明显，可以用 2 点之间的距离和法线方向的夹角度 量. 记顶点 $\boldsymbol{v}_{i}$ 的法向量为 $\boldsymbol{n}_{i}$, 则平滑项定义为

$$
\begin{aligned}
& E_{\text {smo }}\left(l\left(\boldsymbol{v}_{i}\right), l\left(\boldsymbol{v}_{j}\right)\right)= \\
& \quad\left|l\left(\boldsymbol{v}_{i}\right)-l\left(\boldsymbol{v}_{j}\right)\right| \cdot \exp \left(-\beta\left|\boldsymbol{v}_{i}-\boldsymbol{v}_{j}\right|+(1-\beta) \boldsymbol{n}_{i} \cdot \boldsymbol{n}_{j}\right)
\end{aligned}
$$

对于一对拥有不同标签的相邻点，它们的距 离越远，法线夹角越大，越有可能处于关节位置， 则给出一个比较大的惩罚; 如果标签相同，则不做 惩罚. 权重 $\beta$ 用于权衡顶点距离能量和法向夹角 能量, 实验中取 0.05 .

求解式(9)就得到了每个顶点的标签，标签为 0 的顶点即为分割出的局部网格.

综上，本文自动分割局部网格算法的步骤如下.

输人. 源模型 $M$ 和目标模型 $\widetilde{M}$.

输出. 目标模型的局部网格 $\widetilde{M}_{\text {sub }}$.

初始时, 候选点集 $\boldsymbol{C}$ 为空, 待分割点集 $\boldsymbol{U}=\boldsymbol{V}$.

Step1. 对源模型和目标模型做刚性对齐，根据式 (8)计算源模型和目标模型之间的变形程度度量 $M_{\mathrm{D}}$.

Step2. 将待分割点集中 $M_{\mathrm{D}}<0.1$ 的点加人候选点 集 $\boldsymbol{C}$, 即 $\boldsymbol{C}=\left\{\boldsymbol{v}_{i}, M_{\mathrm{D}}\left(\boldsymbol{v}_{i}\right)<0.1, \boldsymbol{v}_{i} \in \boldsymbol{U}\right\}$.

Step3. 找出待分割网格 $U$ 中 $M_{\mathrm{D}}$ 值最大的点 $\boldsymbol{v}_{\max }$, 在候选点集 $C$ 中选取与 $\boldsymbol{v}_{\text {max }}$ 测地线距离最小的点 $\boldsymbol{v}_{\mathrm{key}}$.

Step4. 从 $\boldsymbol{v}_{\text {key }}$ 出发, 分别向 $M_{\mathrm{D}}$ 值最大和最小的方 向搜索点 $\boldsymbol{v}_{p}$ 和 $\boldsymbol{v}_{q}$.

Step5. 计算调和场，依据调和值对目标网格进行 粗略的分割.
Step6. 根据式(9)计算分割的准确边界, 分割出局 部网格 $\widetilde{M}_{\text {sub }}$.

Step7. 更新待分割点集 $\boldsymbol{U}=\boldsymbol{U}-\left\{\boldsymbol{v}_{\mathrm{sub}}, \tilde{\boldsymbol{v}}_{\mathrm{sub}} \in \widetilde{\boldsymbol{M}}_{\mathrm{sub}}\right\}$.

Step8. 若发生变形的局部网格分割完全, 则结束; 否则, 转 Step3.

图 4 所示为进行一次局部网格分割的流程图

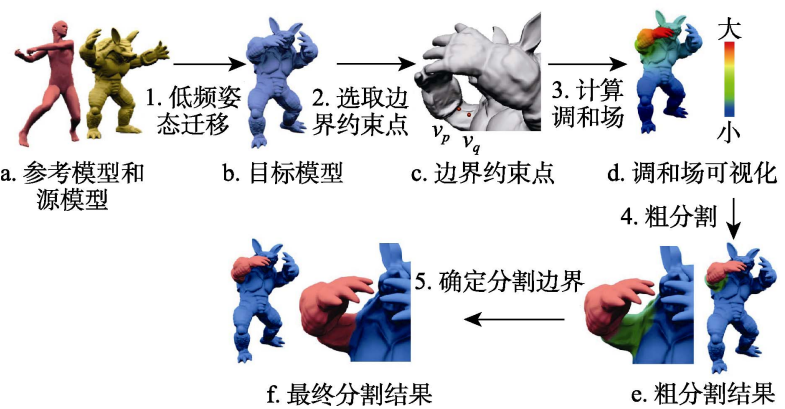

图 4 自动分割局部网格算法的流程

图 5 和图 6 给出 2 个例子, 其中, 图 5a 和图 6a 中红色部分所示为选取的用于计算调和场的边 界约束点; 图 $5 \mathrm{~b}$ 和图 $6 \mathrm{~b}$ 所示为根据调和值进行粗 分割的结果, 红色部分为区域 $A$, 蓝色部分为区 域 $B$, 绿色部分为模糊区域 $C$; 图 $5 \mathrm{c}$ 和图 $6 \mathrm{c}$ 所示 为最终分割结果. 可以看出, 本文方法可以定位到 发生较大变形的局部网格区域, 且能在靠近关节 处将其与整体网格分割.

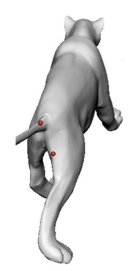

a. 边界约束点

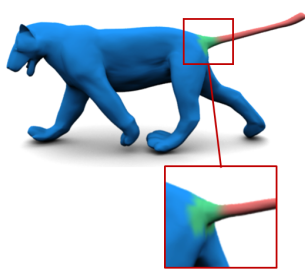

b. 粗分割

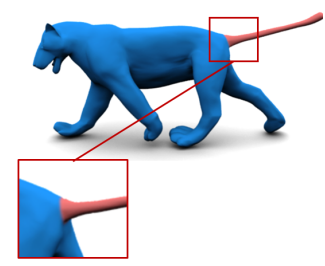

c. 最终分割结果
图 5 狮子模型的分割

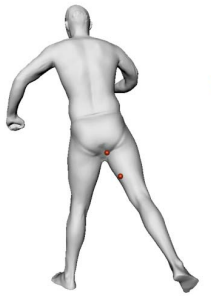

a. 边界约束点

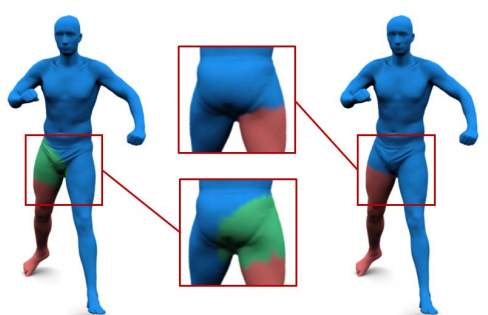

b. 粗分割 c. 最终分割结果
图 6 人体模型的分割

\section{3 分层姿态迁移}

经过前面的步骤得到了参考模型和目标模型 对应的局部网格, 记分割后得到的参考模型 $M^{\prime}$ 的 
局部网格为 $P^{\prime}$, 对应的全局姿态迁移后的目标模 型 $\widetilde{M}$ 的局部网格为 $\widetilde{P}$, 则相对于参考模型 $M^{\prime}$ 的 中频姿态转化为局部网格 $P^{\prime}$ 的低频姿态.

与全局姿态迁移类似，利用基于变形图的谱 姿态迁移技术对 $P^{\prime}$ 和 $\widetilde{P}$ 做姿态迁移, 对所有变形 不充分的局部网格重复这一操作，直到参考模型 所有的姿态被充分迁移.

在对局部网格进行姿态迁移时，为了防止因 全局旋转造成的拟合错误，需要固定局部网格的 部分顶点. 由于局部网格的边界处相对于整体网 格来说是关节位置，一般不会发生改变，因此将局 部网格的边界点作为变形前后的固定点约束，即 在式(5)的基础上, 加上固定点约束能量项

$$
E_{\text {fixed }}=\sum_{\boldsymbol{v}_{i} \in B}\left\|\tilde{\boldsymbol{v}}_{i}-\boldsymbol{v}_{i}\right\|_{2}^{2}
$$

其中, $B$ 为局部网格的边界点的集合; $\tilde{v}_{i}$ 由变形 图节点根据式(2)得到. 综上，对局部网格的姿态 迁移即是求解优化问题

$\min _{\boldsymbol{R}_{0}, \boldsymbol{t}_{0}, \cdots, \boldsymbol{R}_{m-1}, \boldsymbol{t}_{m-1}} w_{\text {freq }} E_{\text {freq }}+w_{\text {rot }} E_{\text {rot }}+w_{\text {reg }} E_{\text {reg }}+w_{\text {fixed }} E_{\text {fixed }}$.

实验中， $w_{\text {fixed }}=6$, 其余权重取值参照第 2.3 节.

虽然对边界点的位置做了约束，但解优化问 题时需要综合考虑各约束项的影响, 最终求解出 来的目标网格的边界点与源网格可能不完全一致, 直接拼接有可能导致拼接处的不自然变形. 为了 使拼接处能够自然过渡, 再次利用变形图对低频 姿态迁移结果进行变形，进而实现局部网格的变 形，而不是将局部网格与整体网格做物理拼接.

图 7 所示为局部网格的姿态迁移结果，红色区 域代表进行中频姿态迁移的局部网格. 其中，图 $7 \mathrm{a}$ 所示为 Yin 等 ${ }^{[6]}$ 方法结果，从上到下分别为全局 低频姿态迁移、局部网格的姿态迁移，可以看出局 部网格在姿态迁移前后的朝向发生了改变; 而图 $7 \mathrm{~b}$ 所示本文方法没有发生朝向变化，且拼接处过 渡自然.

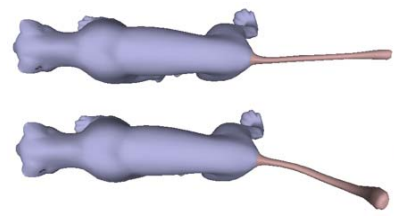

a. Yin 等 ${ }^{[6]}$

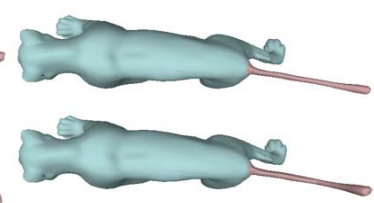

b. 本文
图 7 局部网格的姿态迁移

\section{4 实验结果与分析}

本文实验在配置为 Intel i7 CPU, 8 GB 内存,
NVIDIA GTX 1060 显卡的 PC, Windows 10 操作系 统上用 $\mathrm{C}++$ 和 Matlab 实现. 本节从主观视觉和客 观数值上将 Yin 等 ${ }^{[6]}$ 方法和本文方法的结果进行对 比与分析.

\section{1 姿态迁移结果展示}

表 1 给出每个实验示例的简单介绍，示例名称 “A-B”表示由参考模型 $A$ 向源模型 $B$ 迁移. 图 8 图 12 所示为分层姿态迁移的结果.

表 1 实验示例介绍

\begin{tabular}{lccl}
\hline 示例名称 & 对应图例 & 连接关系 & 对象 \\
\hline 马-马 & 图 8 & 相同 & 相同 \\
猫-猫 & 图 9 & 不同 & 相同 \\
猫-狮子 & 图 10 & 相同 & 不同 \\
男人-男人 & 图 11 & 不同 & 不同 \\
女人-男人 & 图 12 & 不同 & 不同 \\
\hline
\end{tabular}

(1) 相同连接关系的同一对象

图 8 所示为 2 个相同连接关系、不同姿态的马 模型之间的姿态迁移，Yin 等 ${ }^{[6]}$ 方法结果已经相当 接近参考模型的姿态, 但马的左后腿相较于源模 型出现前伸的情况, 右后腿弯曲程度不足, 尾巴末 端有轻微的弯曲变形, 而本文方法结果更接近参 考模型. 此外, 本文只进行了低频姿态迁移就能达 到这样的效果，而 Yin 等 ${ }^{[6]}$ 方法还对马模型的右后 腿、尾巴和头部做了姿态迁移.

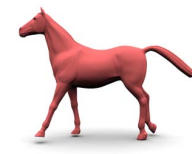

a. 参考模型

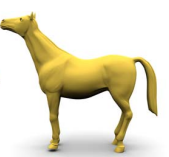

b. 源模型

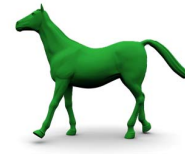

c. Yin 等 $[6]$

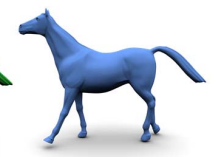

d. 本文
图 8 相同连接关系的同一对象之间的姿态迁移

(2) 不同连接关系的相同对象

图 9 中 2 个拓扑结构不同的猫模型具有相似的 表面形状，Yin 等 ${ }^{[6]}$ 方法结果中猫尾巴弯曲较生硬, 末端变粗，后腿有向后伸展的趋势，而本文方法结 果中猫尾巴的弯曲轨迹更自然平滑, 形状与源模 型更加接近，网格的质量也更好.

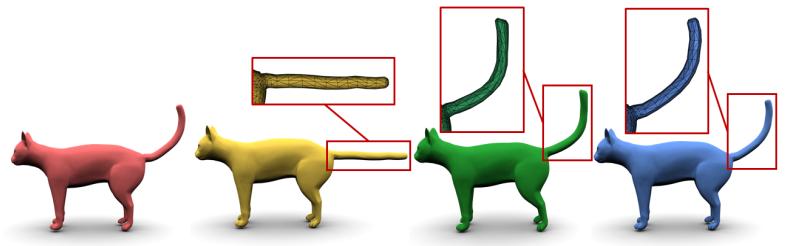

$\begin{array}{lll}\text { a. 参考模型 } & \text { b. 源模型 } \quad \text { c. Yin 等 } \\ & {[6]} & \text { d. 本文 }\end{array}$

图 9 不同连接关系的相同对象之间的姿态迁移 
(3) 相同连接关系的不同对象

图 10 中的猫和狮子模型具有相同的拓扑结构, 但表面形状差异很大. 在这个例子中, 对整体姿态 进行迁移之后，将尾巴部分分割出来做第 2 层次的 姿态迁移. Yin 等 ${ }^{[6]}$ 方法结果中狮子尾巴的末端明 显变粗，而本文方法结果能保持源模型的形状.

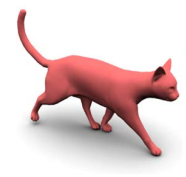

a. 参考模型

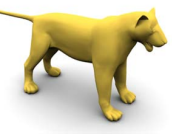

b. 源模型

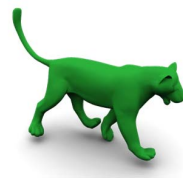

c. Yin 等 ${ }^{[6]}$

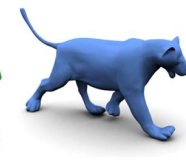

d. 本文
图 10 相同连接关系的不同对象之间的姿态迁移

(4) 不同连接关系的不同对象

图 11 和图 12 所示为一种更为普遍的情况, 参 考模型和源模型的对象和连接关系都不相同. 图 11 中, Yin 等 ${ }^{[6]}$ 的结果双腿张开的距离和弯曲程度、 左小臂的弯曲角度都比参考模型的大，相比之下 本文结果更接近参考模型的姿态. 此外, Yin 等 ${ }^{[6]}$ 方 法需要对双手、双腿和头部都进行分层姿态迁移, 而本文方法在全局低频姿态迁移之后，双手的姿 态已经迁移到位 (见图 3), 只需对双腿和头部迁移. 图 12 的例子是将女性人体模型的姿态迁移到男性 人体模型上，Yin 等 ${ }^{[6]}$ 方法结果中右小臂的朝向更 接近参考模型, 但与源模型相比, 双手手臂均变得 更纤细了，而本文方法结果对于手臂的形状保持 效果更好，姿态的迁移结果也在可以接受的范围.

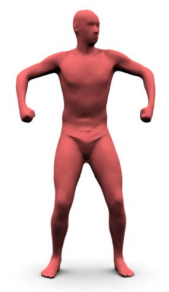

a. 参考模型

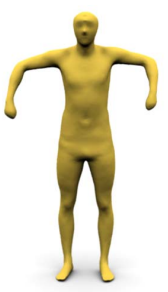

b. 源模型

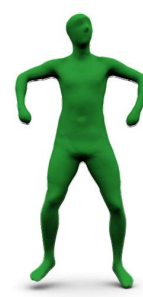

c. Yin 等 ${ }^{[6]}$

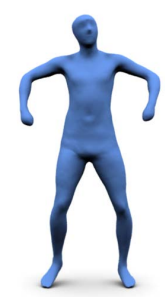

d. 本文
图 11 不同连接关系的人体模型之间的姿态迁移 1

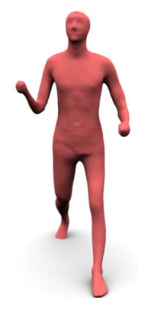

a. 参考模型

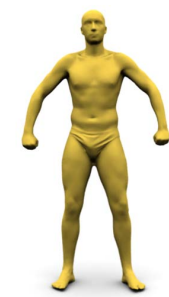

b. 源模型

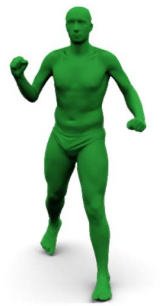

c. Yin 等 ${ }^{[6]}$



d. 本文
图 12 不同连接关系的人体模型之间的姿态迁移 2

\section{2 数值分析}

4.2.1 次级姿态迁移的次数统计

在全局的低频姿态迁移后，对一个局部模型 的姿态迁移记为一次次级姿态迁移. 表 2 给出 Yin 等 ${ }^{[6]}$ 方法和本文方法所需要的次级姿态迁移次数 和迁移部位. 由于变形图局部控制的特性, 因此局 部的变形不受距离其过远的顶点的影响, 部分局 部网格的姿态在低频迁移阶段可以得到较充分的 迁移. 当中频姿态较丰富时, 本文方法能在一定程 度上减少分层姿态迁移的次数.

表 2 不同方法次级姿态迁移的迁移次数 (迁移部位)对比

\begin{tabular}{lll}
\hline \multicolumn{1}{c}{ 示例 } & \multicolumn{1}{c}{ Yin 等 $^{[6]}$} & \multicolumn{1}{c}{ 本文 } \\
\hline 马-马 & 3(尾巴, 右后腿, 头) & 0 \\
猫-猫 & 1(尾巴) & 1(尾巴) \\
猫-狮子 & 1(尾巴) & 1(尾巴) \\
男人-男人 & 5(左、右臂, 左、右腿, 头) & 3(左、右腿, 头) \\
女人-男人 & 3(左、右臂, 右腿) & 3(左、右臂, 右腿) \\
\hline
\end{tabular}

\subsection{2 平均距离误差}

对姿态迁移结果做定量比较，在消除整体平 移和旋转引起的差异后, 计算目标模型与基准模 型逐点欧几里得度量的平均值, 用这个平均值与 包围盒对角线长度的比值作为误差. 示例 “马-马” 中的参考模型与源模型是同构对象的不同姿态, 直接使用参考模型作为基准模型. “猫-猫”示例中 的参考模型是与源模型同构的模型的一个采样, 使用采样前的参考模型作为基准模型. 表 3 给出这 2 个例子的误差, 数据表明, 本文方法结果更接近 参考模型.

表 3 不同方法平均距离误差对比

\begin{tabular}{cccc}
\hline 示例 & 顶点数/面数 & Yin 等 ${ }^{[6]}$ & 本文 \\
\hline 马-马 & $8431 / 16858$ & 1.3174 & 1.1664 \\
猫-猫 & $7207 / 14410$ & 1.2645 & 0.0424 \\
\hline
\end{tabular}

\subsection{3 表面积和体积的变化}

为了证明本文方法在形状保持上的优越性, 对姿态迁移前后网格的表面积和体积的变化进行 对比, 计算目标网格的表面积和体积相对于源网 格变化的绝对值百分比. 图 $13 \mathrm{a}$ 和图 $13 \mathrm{~b}$ 分别为表 面积和体积的变化, 蓝色和橙色的柱状条分别代 表 Yin 等 ${ }^{[6]}$ 方法和本文方法的结果. 由于局部刚性 变化和相邻顶点连续变化的约束, 本文使用变形 


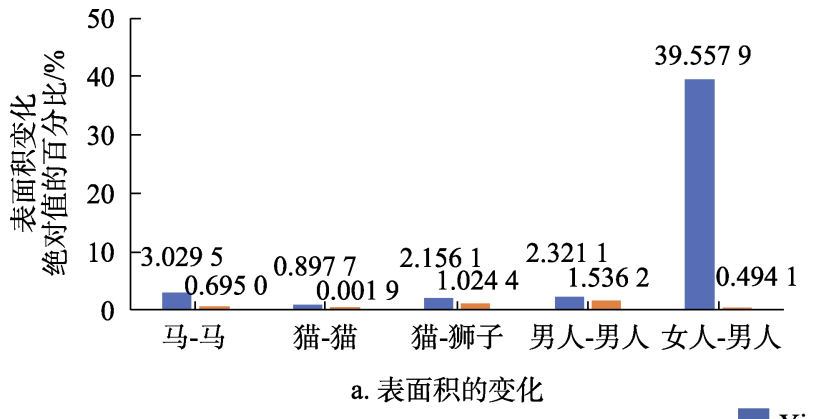

Yin 等[6];

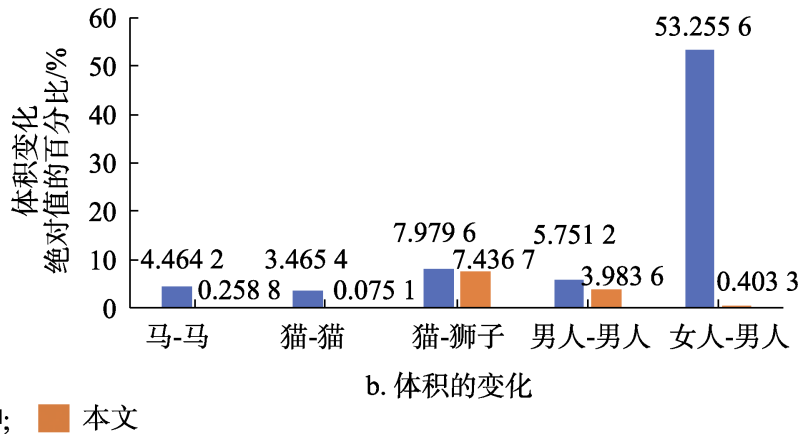

图 13 姿态迁移前后网络的表面积、体积的变化

图的姿态迁移方法在变形前后网格的表面积和体 积变化幅度较小, 能够较好地保持网格的形状.

\subsection{4 时间性能}

表 4 给出所有示例所使用的参考模型、源模型 和变形图的规模, 以及测地线距离、耦合准调和基 的计算、全局和次级姿态迁移这几个关键步骤的耗
时. 其中, 计算测地线距离的耗时较多. 此外, 由 于低频系数能量项涉及源模型的所有顶点, 因此当 变形图规模比较大时, 求解的时间也相对较长. 与 文献[6]相比，本文方法虽然牺牲了一定的效率，但 是提高了模型形状的保持质量, 自动分层的技术也 能减少人工操作的耗时, 总体来说能够令人满意.

表 4 本文实验示例耗时

\begin{tabular}{lllccccc}
\hline \multicolumn{1}{c}{ 示例 } & 参考模型 $(\mathrm{V} / \mathrm{F})$ & 源模型 $(\mathrm{V} / \mathrm{F})$ & 变形图 $(\mathrm{V} / \mathrm{F})$ & 测地线距离 & 耦合准调和基 & 全局姿态迁移 & 次级姿态迁移 \\
\hline 马-马 & $8431 / 16858$ & $8431 / 16858$ & $352 / 700$ & 227.69 & 9.37 & 99.33 & \\
猫-猫 & $3604 / 7204$ & $7207 / 14410$ & $319 / 634$ & 160.35 & 8.58 & 26.52 & 28.60 \\
猫-狮子 & $7207 / 14410$ & $7207 / 14410$ & $472 / 936$ & 159.96 & 14.58 & 165.91 & 25.02 \\
男人-男人 & $6241 / 12478$ & $6449 / 12894$ & $645 / 1286$ & 124.40 & 11.86 & 251.18 & 83.63 \\
女人-男人 & $6442 / 12880$ & $7506 / 15008$ & $489 / 974$ & 183.29 & 10.71 & 156.92 & 192.40 \\
\hline
\end{tabular}

注. $\mathrm{V}$ 表示顶点数, $\mathrm{F}$ 表示面片数.

\section{5 结 语}

本文研究 2 个模型之间的姿态迁移问题，主要 工作包括以下 3 个方面.

(1) 提出一个自动化检测并分割局部网格的 方法. 根据变形前后三角形面片的变形梯度和顶 点偏移量自动检测出发生较大变形的部位, 并定 位到期望分割的区域; 然后根据期望的分割部位 求解拉普拉斯方程得到每个顶点的调和值，根据 调和值将网格粗略分割; 最后利用最小割算法在 模糊区域中计算出准确的分割边界，使分割边界 落在关节区域. 该方法能够将变形程度大的局部 网格分割出来，以便进行下一层次的姿态迁移，可 以减少一定的人工交互操作.

(2) 提出变形图驱动的谱姿态迁移方法, 根据 拉普拉斯矩阵的低频成分编码模型的整体姿态的 特性，通过约束参考模型和源模型基于耦合准调 和基的低频系数相等来实现姿态迁移; 同时，通过 约束顶点的刚性变化和相邻顶点间变化的连续性
保持模型表面的细节，使变形前后的网格具有良 好的形状保持能力; 此外, 变形图局部控制的特性 能在一定程度上能够减少次级姿态迁移的次数.

(3) 提出优化局部网格姿态迁移和拼接的方 法. 对局部网格的姿态迁移同样使用基于变形图 的方法, 通过固定边界点不动来避免发生全局旋 转, 该方法不会导致局部网格的朝向发生改变; 在 将局部网格与整体网格拼接时, 摒弃了直接物理 拼接的方法, 改为对低频迁移结果进行局部变形 以获取局部网格的姿态，一是可以保证交界处的自 然过渡, 二是从理论上来说, 对局部网格分别进行 姿态迁移再拼接可能会导致整体存在割裂感，整体 变形的方法能实现一个整体联动的变化, 克服各部 分单独迁移的割裂感，获得自然的迁移结果.

为了充分说明本文方法的有效性，本文从视 觉感受和数值分析方面对实验结果做了分析, 并 与现有的姿态迁移工作进行对比. 实验结果表明, 本文方法能够产生合理的姿态迁移结果, 且能更 好地保持模型的形状，同时减少了整个处理流程 
中的交互操作.

然而，本文工作还是存在一定的问题. 自动检 测局部网格的方法是基于网格的变形特征，换言 之，如果局部网格在低频姿态迁移阶段没有发生 变化或变化程度很小, 而该局部网格又需要进行 次级姿态迁移, 那么本文方法无法对其自动分割, 此时必须引人人工操作。未来希望可以找到一种 完全自动的方法, 可以通过比较目标模型与参考 模型姿态的差异性定位姿态迁移不充分的局部网 格并对其分割. 对于人体模型间的姿态迁移，可以 考虑提取骨架来进行差异化分析，或者在姿态迁 移前预先对参考模型和源模型进行刚性块共分割; 然后逐个比较刚性块间的姿态差异性，判断该局 部块是否需要进行次级姿态迁移. 另外, 变形图的 构造需要计算顶点间的测地线距离作为权重，这 是一项耗时的操作; 求解变形图的优化问题的时 间也比较长, 未来可以利用 GPU 进行加速, 进一 步提升本文方法的性能.

\section{参考文献(References):}

[1] Gain J, Bechmann D. A survey of spatial deformation from a user-centered perspective[J]. ACM Transactions on Graphics, 2008, 27(4): Article No.107

[2] Sumner R W, Popovic J. Deformation transfer for triangle meshes[J]. ACM Transactions on Graphics, 2004, 23(3): 399-405

[3] Baran I, Vlasic D, Grinspun E, et al. Semantic deformation transfer[J]. ACM Transactions on Graphics, 2009, 28(3): Article No.36

[4] Levy B. Laplace-Beltrami eigenfunctions towards an algorithm that "understands" geometry[C] //Proceedings of the IEEE International Conference on Shape Modeling and Applications. Los Alamitos: IEEE Computer Society Press, 2006: 66-73

[5] Kovnatsky A, Bronstein M M, Bronstein A M, et al. Coupled quasi-harmonic bases[J]. Computer Graphics Forum, 2013, 32(2pt4): 439-448

[6] Yin M X, Li G Q, Lu H N, et al. Spectral pose transfer[J]. Computer Aided Geometric Design, 2015, 35/36(SI): 82-94

[7] Barr A H. Global and local deformations of solid primitives[J]. Computer Graphics, 1984, 18(3): 21-30

[8] Sederberg T W, Parry S R. Free-form deformation of solid geometric models[C] //Proceedings of the 13th Annual Conference on Computer Graphics and Interactive Techniques. New York: ACM Press, 1986: 151-160

[9] Sumner R W, Schmid J, Pauly M. Embedded deformation for shape manipulation[J]. ACM Transactions on Graphics, 2007, 26(3): Article No.80

[10] Lipman Y, Sorkine O, Cohenor D, et al. Differential coordinates for interactive mesh editing[C] //Proceedings of the Shape Modeling International. New York: ACM Press, 2004
181-190

[11] Sorkine O, Alexa M. As-rigid-as-possible surface modeling[C] //Proceedings of the 5th Eurographics Symposium on Geometry Processing. Aire-la-Ville: Eurographics Association Press, 2007: 109-116

[12] Frohlich S, Botsch M. Example-driven deformations based on discrete shells[J]. Computer Graphics Forum, 2011, 30(8): 2246-2257

[13] Su Peng, Yin Mengxiao, Wang Yupan, et al. Spectral pose transfer based on deformation graph[J]. Journal of Graphics, 2019, 40(2): 282-289(in Chinese) (苏鹏，尹梦晓，王宇攀，等. 基于变形图的谱姿态迁移 [J]. 图学学报, 2019, 40(2): 282-289)

[14] Wang Lin. Research and implementation of spectral pose transfer on polygon mesh[D]. Nanning: Guangxi University, 2017(in Chinese)

(王淋. 谱姿态迁移在多边形网格模型上的研究与实现 $[D]$. 南宁: 广西大学, 2017)

[15] Yin Mengxiao, Wang Lin, Zhong Cheng, et al. Low-frequency spectral pose transfer between quad mesh and triangle mesh[J]. Journal of Guangxi University: Natural Science Edition, 2019, 44(3): 703-711(in Chinese)

(尹梦晓，王淋，钟诚，等. 四边形网格与三角形网格模型间 的低频谱姿态迁移 [J]. 广西大学学报: 自然科学版, 2019, 44(3): 703-711)

[16] Lifkooee M Z, Liu C, Liang Y, et al. Real-time avatar pose transfer and motion generation using locally encoded Laplacian offsets[J]. Journal of Computer Science and Technology, 2019, 34(2): 256-271

[17] Basset J, Wuhrer S, Boyer E, et al. Contact preserving shape transfer: retargeting motion from one shape to another[J]. Computers \& Graphics, 2020, 89: 11-23

[18] Katz S, Tal A. Hierarchical mesh decomposition using fuzzy clustering and cuts $[\mathrm{J}]$. ACM Transactions on Graphics, 2003, 22(3): 954-961

[19] Boykov Y, Kolmogorov V. An experimental comparison of min-cut/max-flow algorithms for energy minimization in vision[J]. IEEE Transactions on Pattern Analysis and Machine Intelligence, 2004, 26(9): 1124-1137

[20] Liu R, Zhang H. Segmentation of 3D meshes through spectral clustering[C] //Proceedings of the 12th Pacific Conference on Computer Graphics and Applications. Los Alamitos: IEEE Computer Society Press, 2004: 298-305

[21] Ji Z P, Liu L G, Chen Z G, et al. Easy mesh cutting[J]. Computer Graphics Forum, 2006, 25(3): 283-291

[22] Fan L B, Liu L G, Liu K. Paint mesh cutting[J]. Computer Graphics Forum, 2011, 30(2): 603-612

[23] Zheng Y Y, Tai C L. Mesh decomposition with cross-boundary brushes[J]. Computer Graphics Forum, 2010, 29(2): 527-535

[24] Meng M, Fan L B, Liu L G. iCutter: a direct cut-out tool for 3D shapes[J]. Computer Animation \& Virtual Worlds, 2011, 22(4): 335-342

[25] Fan L B, Meng M, Liu L G. Sketch-based mesh cutting: a comparative study[J]. Graphical Models, 2012, 74(6): 292-301.

[26] James D L, Twigg C D. Skinning mesh animations[J]. ACM Transactions on Graphics, 2005, 24(3): 399-407 
[27] Comaniciu D, Meer P. Mean shift: a robust approach toward feature space analysis[J]. IEEE Transactions on Pattern Analysis and Machine Intelligence, 2002, 24(5): 603-619

[28] de Aguiar E, Theobalt C, Thrun S, et al. Automatic conversion of mesh animations into skeleton-based animations $[\mathrm{J}]$. Computer Graphics Forum, 2008, 27(2): 389-397

[29] Le B H, Deng Z G. Robust and accurate skeletal rigging from mesh sequences[J]. ACM Transactions on Graphics, 2014, 33(4): Article No.84

[30] Le B H, Deng Z G. Smooth skinning decomposition with rigid bones[J]. ACM Transactions on Graphics, 2012, 31(6): Article No.199

[31] Hasler N, Thormahlen T, Rosenhahn B, et al. Learning skeletons for shape and pose[C] //Proceedings of the ACM SIGGRAPH symposium on Interactive 3D Graphics and Games. NewYork: ACM Press, 2010: 23-30

[32] Wuhrer S, Brunton A. Segmenting animated objects into near-rigid components[J]. The Visual Computer, 2010, 26(2):
147-155

[33] Li Guiqing, Ma Weiyin, Bao Hujun. Fitting system using Loop subdivision surfaces with sharps features[J]. Journal of Computer-Aided Design \& Computer Graphics, 2005, 17(6): 1179-1185(in Chinese)

(李桂清, 马维银, 鲍虎军. 带尖锐特征的 Loop 细分曲面拟 合系统 $[\mathrm{J}]$. 计算机辅助设计与图形学学报, 2005, 17(6): 1179-1185)

[34] Garland M, Heckbert P S. Surface simplification using quadric error metrics[C] //Proceedings of the 24th Annual Conference on Computer Graphics and Interactive Techniques. New York: ACM Press, 1997: 209-216

[35] Moenning C, Dodgson N A. Fast marching farthest point sampling for point clouds and implicit surfaces[R]. Cambridge: Cambridge University, 2003

[36] Goodall C. Procrustes methods in the statistical analysis of shape[J]. Journal of the Rroyal Statistical Society Series B: Methodological, 1991, 53(2): 285-321 